\title{
THz Signal Denoising via Redundant Representation
}

\author{
S.M. Zhu, B.W.-H. Ng, B.M. Fischer and D. Abbott \\ Centre for Biomedical Engineering (CBME) and School of Electrical \& Electronic Engineering, \\ The University of Adelaide 5005, Australia
}

\begin{abstract}
Denoising is widely used in $\mathbf{T H z}$ signal processing. In recent years, there have been many papers about applying wavelet-based denoising techniques to achieve clean THz signal. Traditionally, denoising by shrinkage on orthogonal, decimated wavelet transforms satisfies most THz applications with dramatic improvements in SNR for TDS pulses. In this paper, we apply the shrinkage technique to non-orthogonal, redundant THz signal representations to TDS pulses and it is shown that this scheme is capable of even an even greater SNR gains.
\end{abstract}

\section{INTRODUCTION AND BACKGROUND}

$\mathrm{THz}$ radiation is loosely defined as having a frequency range of 0.1 to $10 \mathrm{THz}$, which is situated between the frequency ranges of microwave radiation and infrared light. Until the $\mathrm{THz}$ time-domain spectroscopic (THz-TDS) technique was invented, research in this area was very limited due to high atmospheric absorption. After the 1980's, THz-TDS received a great deal of attention thanks to broad applicability to detection of biological and chemical materials, quality control, gas identification and T-ray imaging [7-11]. However, some of these applications, such as those in security, need a fast scanning system to acquire data quickly, usually at the expense of sacrificing signal quality. Such noisy datasets make analysis difficult and the results are not always reliable. Thus, denoising techniques are often used after signal acquisition to accommodate subsequent processing.

Shrinkage techniques [2] were introduced by Donoho and Johnstone in 1994, and they have been widely and effectively applied for denoising signals which exhibit sparsity in some non-redundant representation. Its first application in $\mathrm{THz}$ appeared in a paper on gas sensing [9]. Ferguson et al [4] studied this technique in detail, using additive Gaussian noise to model experimental imperfections, and it was shown that shrinkage techniques succeeded in achieving a very satisfying result in removing the Gaussian noise. Meanwhile, Lang et al. [6] invented a novel approach for the reduction of noise based on the traditional wavelet transformation by employing shrinkage on undecimated and consequently redundant wavelet representations of various idealised signals. This method improved noise reduction dramatically and can be applied to a large class of signals. More recently, Elad [3] has also shown that simple shrinkage works for redundant representations in image denoising experiments with redundant and nonredundant representations. He was able to prove that shrinkage is a special case of an iterative cost minimisation objective. In this paper, we draw inspiration from Lang and Elad's work, and will investigate the use of redundant representations for THz-TDS signals, which offer sufficient sparsity and allow the shrinkage method to work.

\section{A. Discrete Wavelet Transform (DWT) based denoising}

Wavelet transform (WT) uses time-frequency localised basis functions as opposed to Fourier Transform (FT) which used infinite sinusoids as the basis functions. Since wavelets are localised both in time and frequency, they require few coefficients to represent pulse-like signals such as those found in THz TDS. Moreover DWT is very efficient since its wavelet coefficients can be calculated with the computational complicity of $O(N)$. A recursive structure of Filter Bank is utilised to implement a DWT, shown in Figure 1.

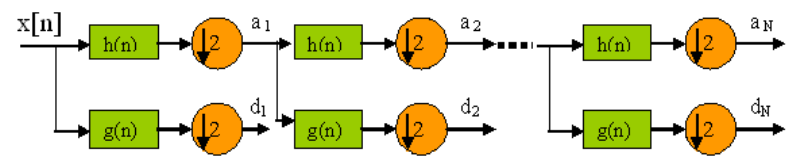

Fig. 1. N Level Filter Bank implementation of DWT. Input signal $x[n]$ flows through low- and high-pass filters before being decimated by 2. $a_{i}$ and $d_{i}$ are approximation and detail coefficients at $i^{\text {th }}$ level of decomposition.

Shrinkage is one of the most commonly used tools in WT domain signal processing. It is widely used for denoising. There are two types of thresholding used as part of shrinkage: hard and soft. Hard thresholding sets a coefficient value $x_{i}$ to zero if it is below $T_{n}$, where $T_{n}$ is some estimated threshold value.

$$
y_{i}=\left\{\begin{array}{lr}
x_{i} & \left|x_{i}\right| \geq T_{n} \\
0 & 0 \leq T_{n}
\end{array}\right.
$$

We use a Gaussian white additive noise model in this paper. The noisy signal can be expressed as $y=x+n$, where is a noise-free signal and is the additive noise. Hard thresholding is performed via the following steps:

1) Calculate wavelet coefficients $d_{i}$ by applying DWT.

2) Estimate the threshold value $T_{n}$

$$
T_{n}=\sigma \sqrt{2 \log (N)}
$$

where $\mathrm{N}$ is the signal length and $\sigma$ is standard deviation of noise at each decomposition level, estimated by

$$
\sigma=\frac{M A D}{0.6745}
$$


where MAD represents median absolute value of detail coefficients.

3) Perform shrinkage

4) Reconstruct the signal using the shrunk (detail only) coefficients through inverse DWT

\section{B. Undedicated Discrete Wavelet Transform (UDWT)}

In DWT, after each filtering step, the output is downsampled by two, which effectively removes the redundant information and keep the data size constant. The advantage is less computation and lower memory requirements to store intermediate value during the transform. However, this type of transform is shift variant $[1,6]$; there are no obvious relationships between the wavelet coefficients of a signal and its shifted versions. Many research groups independently discovered the UDWT by eliminating the downsampling to achieve shift variance, at the expense of data redundancy. Figure 2 illustrates the Filter Bank implementation of UDWT. Denoising procedure of UDWT is identical to DWT except for the transform.

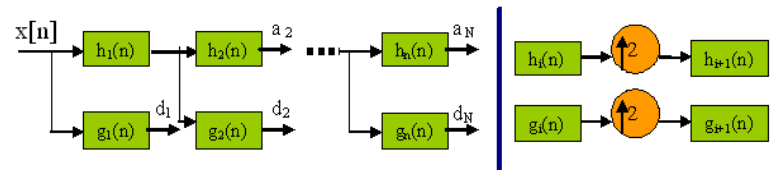

Fig. 2. N Level Filter Bank implementation of UDWT. Input signal $x[\mathrm{n}]$ flows through low- and high-pass filters without being decimated. $a_{i}$ and $d_{i}$ are approximation and detail coefficients at $i^{\text {th }}$ level of decomposition. Each level's filters are upsampled versions of the previous.

\section{RESULTS}

In our first denoising experiments, white noise was added to the original signal, a reference $\mathrm{THz}$ pulse, such that the SNR is variable in order to simulate various levels of measurement errors. This reference pulse is from the paper of $\mathrm{THz}$ application in chemical recognition [5] where the details of system setup and experiment parameters are described. The signals were denoised 100 times to average the different noise realizations using two methods. We applied Daubechies wavelet with order 5 in the denoising process. Other experimental parameters are: signals are of length 512, the decomposition depth scales is 8 and hard thresholding is chosen. In Figure 3, it is obvious that UDWT outperforms DWT by $6 \mathrm{~dB}$ with the SNR in input in the range of $-5 \mathrm{~dB}$ to $10 \mathrm{~dB}$. In a sense of visualization, Figure 4 shows a smoother denoised signal (in red color) as opposed to artifacts-introduced denoised signal (in green).

\section{CONCLUSION}

From the results, it is clear that there is a SNR gain of up to $6 \mathrm{~dB}$ from a redundant wavelet transform over existing non-redundant versions. This method can be interpreted as the repetition of Donoho shrinkage technique on shifted versions of original signal. It was found that, in terms of both SNR andvisual results, UDWT-based denoising outperforms DWTs. These improvements will serve further processing such as any applications to classification.

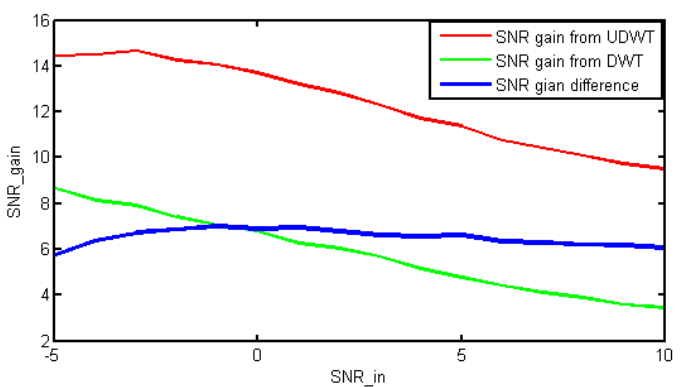

Fig. 3. Denoising performance comparison. SNR_in is the SNR of noisy signal with various realisations. SNR_gain is the SNR gain resulting from UDWT and DWT based denoising according to SNR_in. Both are in dB. Green line measures the SNR after DWT-based denoising; red line is for UDWT-based denoising. Blue line is the SNR gain difference between those two methods.

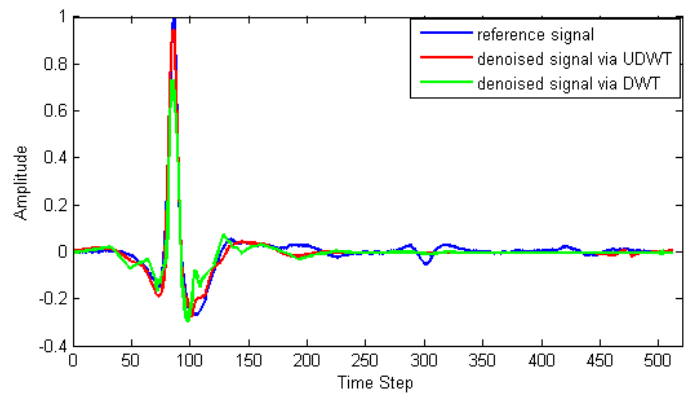

Fig. 4. Visual view of denoised signals. Red line is the denoising result from an input signal with SNR of $3 \mathrm{~dB}$ via UDWT denoising; green line is the result from DWT denoising.

\section{REFERENCES}

[1] R. R. Coifman and D. L. Donoho, "Translation-invariant de-noising," Tech. Rep., 1994.

[2] D. Donoho, "De-noising by soft-thresholding," Information Theory, IEEE Transactions on, vol. 41, no. 3, pp. 613-627, May 1995.

[3] M. Elad, "Why simple shrinkage is still relevant for redundant representations?" IEEE Transactions on Information Theory, vol. 52, no. 12, pp. 5559-5569, 2006.

[4] B. Ferguson and D. Abbott, "Wavelet de-noising of optical terahertz pulse imaging data," Fluctuation and Noise Letters, vol. 1, no. 2, pp. 65-69, 2001.

[5] B. M. Fischer, M. Hoffmann, H. Helm, G. Modjesch, and P. U. Jepsen, "Chemical recognition in terahertz time-domain spectroscopy and imaging," Semicond. Sci. Technol., vol. 20, pp. 246-253, 2005.

[6] H. Guo, M. Lang, J. E. Odegard, and C. S. Burrus, "Nonlinear processing of a shift-invariant DWT for noise reduction and compression," in Proceedings of the International Conference on Digital Signal Processing, Limassol, Cyprus, 26-28 1995, pp. 332-337.

[7] B. B. Hu and M. C. Nuss, "Imaging with terahertz waves," Opt. Lett., vol. 20, no. 16, p. 1716, 1995.

[8] K. Kawase, Y. Ogawa, Y. Watanabe, and H. Inoue, "Non-destructive terahertz imaging of illicit drugs using spectral fingerprints," Opt. Express, vol. 11, no. 20, pp. 2549-2554, 2003.

[9] D. M. Mittleman, R. H. Jacobsen, R. Neelamani, R. G. Baraniuk, and M. C. Nuss, "Gas sensing using terahertz time-domain spectroscopy," Applied Physics B: Lasers and Optics, vol. 67, pp. 379-390, Sep. 1998.

[10] W. R. Tribe, D. A. Newnham, P. F. Taday, and M. C. Kemp, "Hidden object detection: security applications of terahertz technology," in SPIE Conference Series, R. J. Hwu, Ed., vol. 5354, Apr. 2004, pp. 168-176.

[11] W. Withayachumnankul, G. Png, X. Yin, S. Atakaramians, I. Jones, H. Lin, S. Y. Ung, J. Balakrishnan, B.-H. Ng, B. Ferguson, S. Mickan, B. Fischer, and D. Abbott, "T-ray sensing and imaging," Proceedings of the IEEE, vol. 95, no. 8, pp. 1528-1558, Aug. 2007. 